\title{
Missed Opportunities for Transitioning to Oral Antibiotic Therapy
}

\author{
Rebecca G Same, MD¹, Pranita D Tamma, MD, MHS*
}

'Division of Pediatric Infectious Diseases, Department of Pediatrics, Washington University School of Medicine, St Louis, Missouri; ${ }^{2}$ Division of Pediatric Infectious Diseases, Department of Pediatrics, Johns Hopkins University School of Medicine, Baltimore, Maryland.

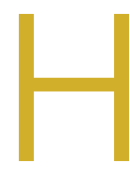

istorically, bacterial infections in hospitalized children were treated with intravenous (IV) antibiotics for the duration of therapy_frequently with placement of a vascular catheter. Risks associated with vascular catheters and the limitations they impose on a child's quality of life are increasingly being recognized-including thrombi, catheter dislodgement, and secondary infections as catheters provide a portal of entry for bacteria into the bloodstream (ie, catheter-associated bloodstream infections) or along the catheter wall (ie, phlebitis). This potential for harm underscores the importance of transitioning to oral antibiotic therapy whenever possible.

In this issue of the Journal of Hospital Medicine, Cotter et al used an administrative database to investigate opportunities to transition from IV to oral antibiotics for patients across multiple pediatric hospitals. ${ }^{1}$ Their novel metric, "percent opportunity," represents the percent of days that there was the opportunity to transition from IV to oral antibiotics. They found that over $50 \%$ of the time, IV antibiotics could have been switched to equivalent oral agents. Furthermore, there was wide variability across institutions in IV-to-oral transitioning practices; $45 \%$ of the variation was seemingly attributable to institution-level preferences.

The large sample size and multicenter nature of this study improve its external validity. However, using administrative data to make assumptions about clinical decision-making has limitations. The definition of opportunity days assumes that any day a child receives other enteral medications provides an "opportunity" to prescribe oral antibiotics instead. This does not account for other reasonable indications to continue IV therapy (eg, endocarditis) and may overestimate true opportunities for conversion to oral therapy. Alternatively, their conservative approach of excluding days when a child received

*Corresponding Author: Pranita D Tamma, MD, MHS; Email: ptamma1@jhmi.edu; Telephone: 410-614-1492.

Received: October 17, 2020; Revised: November 3, 2020;

Accepted: November 4, 2020

๑) 2021 Society of Hospital Medicine DOI 10.12788/jhm.3564 both IV and oral antibiotics may underestimate opportunities for oral transition. Regardless of the precision of their estimates, their findings highlight that there is room to improve the culture of transitioning hospitalized children from IV to oral antibiotic therapy.

Admittedly, the evidence for clinically effective conversion to oral therapy in children remains incomplete. Data support oral antibiotics for hospitalized children with pneumonia, cellulitis, pyelonephritis, and osteoarticular infections-even with associated bacteremia. ${ }^{2}$ There is also evidence for successful conversion to oral therapy for complicated appendicitis, retropharyngeal abscesses, mastoiditis, and orbital cellulitis. ${ }^{2}$

The decision to transition to oral therapy does not need to be delayed until the time of hospital discharge because each additional day of IV therapy poses a cumulative risk. Rather, prescribers should apply a structured approach, such as the "Four Moments of Antibiotic Decision Making," on a daily basis for every hospitalized child receiving antibiotics to prompt timely decisions about discontinuing IV therapy, narrowing IV therapy, or transitioning from IV to oral antibiotic therapy. ${ }^{3}$ We applaud Cotter et al for shedding light on an area in need of standardization of care, which could optimize patient outcomes and minimize harm for a large number of children. ${ }^{1}$ The "percent opportunity" to switch from IV to oral antibiotic therapy is a promising antibiotic stewardship metric, and its association with clinical outcomes merits further investigation.

Disclosures: The authors have nothing to disclose.

\section{References}

1. Cotter JM, Hall M, Girdwood ST, et al. Opportunities for stewardship in the transition from intravenous to enteral antibiotics in hospitalized pediatric patients. J Hosp Med. 2021;16:XXX-XXX. https://doi.org/10.12788/jhm.3538

2 McMullan BJ, Andresen D, Blyth CC, et al. Antibiotic duration and timing of the switch from intravenous to oral route for bacterial infections in children: systematic review and guidelines. Lancet Infect Dis. 2016;16(8):e139-e152. https://doi.org/ 10.1016/S1473-3099(16)30024-X

3. Tamma PD, Miller MA, Cosgrove SE. Rethinking how antibiotics are prescribed: incorporating the 4 moments of antibiotic decision making into clinical practice. JAMA. 2019;321(2):139-140. https://doi.org/ 10.1001/ jama.2018.19509 